\title{
Scaling Laws of Intermittent Plasma Turbulence in Edge of Fusion Devices
}

\author{
Viacheslav P. BUDAEV ${ }^{1,2)}$, Suguru MASUZAKI ${ }^{2)}$, Tomohiro MORISAKI ${ }^{2)}$, Noriyasu $\mathrm{OHNO}^{3)}$, \\ Nobuyuki ASAKURA $^{4)}$, Shuichi TAKAMURA ${ }^{5)}$, Hiroshi YAMADA ${ }^{2)}$ and Akio KOMORI ${ }^{2)}$ \\ 1) Nuclear Fusion Institute, RRC Kurchatov Institute, 123182, Kurchatov Sq.1, Moscow, Russia \\ 2) National Institute for Fusion Science, Oroshi 322-6, Toki, Gifu 509-5292, Japan \\ 3) Eco Topia Science Institute, Nagoya University, Nagoya 464-8603, Japan \\ 4) JAEA, Naka Fusion Research Establishment Naka-machi, Naka-gun, Ibaraki-ken 311-0193, Japan. \\ 5) Faculty of Engineering, Aichi Institute of Technology, 1247 Yachigusa, Yagusa-cho, Toyota 470-0392, Japan
}

(Received 31 October 2007 / Accepted 15 February 2008)

\begin{abstract}
The high-order structure functions have been analyzed to characterize the edge plasma intermittency in fusion devices. The scaling properties of edge turbulence have shown a strong deviation from a prediction of the Kolmogorov's K41 model. The turbulent fluctuations demonstrate a generalized scale invariance and log-Poisson statistics.

(c) 2008 The Japan Society of Plasma Science and Nuclear Fusion Research
\end{abstract}

Keywords: edge plasma turbulence, intermittency, log-Poisson model, extended self-similarity, multifractal statistics

DOI: $10.1585 /$ pfr.3.S1019

\section{Inroduction}

Incompressible hydrodynamic turbulence is described by Kolmogorov theory [1] (K41) which considers a hierarchical energy cascade and the energy scaling $E(k) \sim k^{-5 / 3}$. At large Reynolds numbers $R e>1$, statistical quasiequilibrium of fluctuations is established in the process of energy transfer from large turbulent eddies towards the smaller ones in the inertial range $l(\eta \ll l \ll L$, where $L$ is the largest eddy/structure scale, $\eta$ dissipation Kolmogorov's scale). Investigation of self-similar turbulence properties resulted in multiplicative hierarchical cascade models [2]: the log-normal model K62, the $\beta$-model, and most favorable the log-Poisson model $[3,4]$. To quantify whether boundary conditions influence the statistical properties in turbulence it was proposed multifractal formalism (see [2]). Intermittency leads to a local breaking of the turbulence homogeneity, when 'active' regions coexist with quasi-laminar ones. The intermittent fluctuations demonstrate non-Gaussian statistics, self-similarity and multifractality. The intermittency is observed in turbulent hydrodynamic fluids [3] and in turbulent magnetized plasmas (see e.g., [5]).

The spectrum of magnetohydrodynamic (MHD) turbulence was first addressed by Iroshnikov and Kraichnan (IK) who considered the turbulent energy cascade affected by a magnetic field (see $[6,7]$ ). The IK model yields $E(k) \sim k^{-3 / 2}$ resulted from Alfven decorrelation effect. The validity of the two phenomenologies (K41 and IK) in MHD turbulence and plasma confined in fusion devices is still under a discussion. Numerical and experimen- tal data indicate that in MHD turbulence the energy transfer occurs predominantly in the field-perpendicular direction [6]. This raises a question whether anisotropy is crucial for the energy cascade, and whether it changes the spectrum of turbulence. The two-dimensional direct numerical simulations (DNS) support the IK picture, while three-dimensional simulations and recent analytical results suggest K41 energy spectra (see [6]). A phenomenology of "intermediate" turbulence by Goldreich and Sridhar [8] (GS95) postulates a balance between K41 and IK energy cascades and accounts for the local anisotropy induced by a magnetic field. The GS95 assumes one-dimension filaments as the most dissipative structures, the same as in hydrodynamic turbulence. Other MHD turbulence models assume the singular structure shape of a current sheet $[6,7]$.

There are numerous experimental observations of the magnetized plasma turbulence that share a lot of features of neutral fluid turbulence including many scales, the cascades, strong mixing, non-linear scalings and so on. Despite equations described neutral fluids and plasmas are different they have the same type of scale invariance (dilatation symmetry, namely, $x \rightarrow \lambda x, \mathrm{t} \rightarrow \lambda^{1-h_{\mathrm{t}}}$, $\left.v \rightarrow \lambda^{h} v\right)$. This common dilatation symmetry is responsible for a common scaling property.

Most favorable log-Poisson model of intermittent turbulence consider anisotropic stochastic cascade and the generalized self-similarity implying the long-range correlations, which drive an anomalous transport (superdiffusion). An experimental analysis of the statistical moments in the frame of the log-Poisson model suggests a description of the transport processes in turbulent plasmas. It 
helps to describe transport scaling law $\left\langle\delta x^{2}\right\rangle \propto \tau^{\alpha}$, where $\left\langle\delta x^{2}\right\rangle$ is a displacement of particles across a magnetic field with time $\tau, \alpha$ is a scaling exponent, $\alpha=1$ corresponds to normal diffusion (Einstein law), $\alpha>1$ superdiffusion, $\alpha<$ 1 subdiffusion. Despite the large amount of experimental data that has been obtained in fusion devices, our understanding of the turbulence and diffusive transport process in magnetized plasmas is still rather limited. In this work we focus on the scale invariance property and scaling laws of the edge plasma turbulence in fusion devices and test analytical fits.

\section{Experimental Data}

We analyze Langmuir probe signals of ion saturation current $I_{\text {sat }}$ that is essentially plasma density. On Large Helical Device [9] $I_{\text {sat }}$ was measured by 16 graphite dometype electrodes (diameter of $1 \mathrm{~mm}$ separated by $6 \mathrm{~mm}$ ) embedded in the divertor plate and by reciprocating probe. On JT-60U tokamak measurements (L-mode) in the SOL has been done by reciprocating Mach probes [10] installed at the low field side (LFS) mid-plane and just below the X-point .On T-10 tokamak [5] $\left(R / r=1.5 \mathrm{~m} / 0.4 \mathrm{~m}, I_{\mathrm{p}}=\right.$ $200 \sim 220 \mathrm{kA}, B=2.2 \sim 2.4 \mathrm{~T}$ ) Langmuir probe (tungsten tips of $0.5 \mathrm{~mm}$ in a diameter and $3 \mathrm{~mm}$ in a length) $I_{\text {sat }}$ was measured at SOL in a steady state of repetitive ohmic discharges with no MHD activity. In the linear divertor plasma simulator NAGDIS-II $\left(n_{\mathrm{e}} \sim 10^{20} \mathrm{~m}^{-3}, T_{\mathrm{e}} \sim 1-3 \mathrm{eV}\right.$, $B=0.25 \mathrm{~T})$ attached and detached plasmas were investigated $[5,11]$.

All signals are digitized with the sampling rate $0.5 \mathrm{MHz}$ in JT-60U and LHD and $1 \mathrm{MHz}$ in other devices, the sample number $10^{5}-10^{6}$. No filtering has been used for the signal analysis. Signals (Fig. 1) have the intermittent behaviour typically observed in edge of fusion devices [5].

Frequency bandwidth of the spectrum is in the range of $\sim 1 \mathrm{kHz}-0.5 \mathrm{MHZ}$ corresponding to the drift-wave turbulence. Power spectra have typically decayed with a frequency exhibiting a complicated frequency dependence without a trivial power-law behavior [5].

Previous studies of edge plasma turbulence in these devices [5,9-11] have revealed radial variations of the turbulence parameters, such as power spectrum, amplitude fluctuations, correlation property, cross-field particle turbulent transport etc. The dominant driving mechanisms of turbulence in these devices could be different. At the same time, common properties of edge turbulence such as intermittence, power laws, a self-similarity, long-range correlation are observed in these devices with different magnetic topology, heating and average parameters (density, electron and ion temperature, electric field) of the core and SOL plasma. Origin of these common features is not revealed. It was mentioned above that a hypothesis of the universal property of turbulence intermittency (e.g., based on the log-Poisson statistics of the process) is discussed in the literature. We analyze the intermittent turbulence sig-

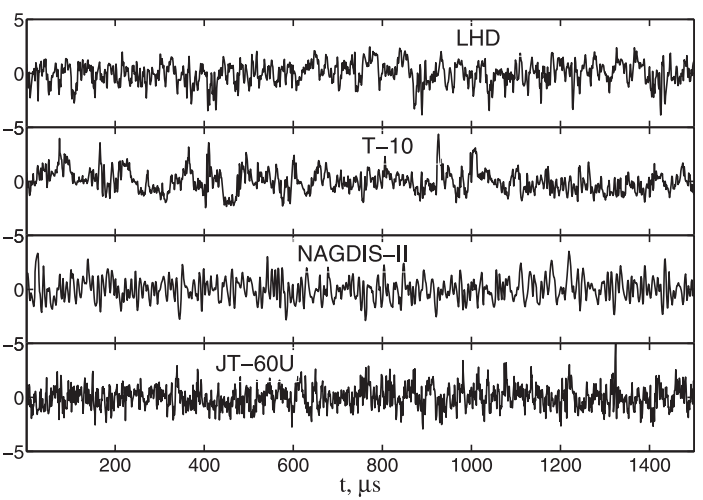

Fig. 1 Time-dependent signals of $I_{\text {sat }}$ subtracted by a mean value and normalized by its standard deviation: LHD (\#10 probe of the divertor plate); the SOL of T-10; the attached plasma in NAGDIS-II at $r=18 \mathrm{~mm}$, the mid-plane LFS SOL of JT-60U at $r=41.8 \mathrm{~mm}$.

nals from the SOL of different fusion devices to illustrate a relevance of this approach for the interpretation of experimental data.

\section{Generalized Scale Invariance}

The classical approach [12] for an exploration of statistical features is an analysis of statistical moments (structure functions) $S_{q}(\tau)=\left\langle|X(t+\tau)-X(t)|^{q}\right\rangle$, where $\langle\cdots\rangle$ means an ensemble average of the time-dependent signal $X(t)$. The structure function technique is equivalent to the detailed investigation of the probability distribution function of the turbulent fluctuation. The Kolmogorov theory K41 of isotropic turbulence infers Gaussian statistics for fluctuations. It predicts the structure function scaling $S_{q}(\tau) \sim \tau^{\zeta(q)}, \zeta(q)=q / 3$, in the inertial range. Experimental investigations of the developed hydrodynamic turbulence demonstrate a departure of the high-order structure function scalings $(q>3)$ from the Kolmogorov K41 prediction due to the intermittency. The experimental structure functions typically shows a power law only over a limited (inertial) range $\eta \ll l \ll L$ (of $\sim 10 \mu$ s in Fig. 2). Whereas the generalized self-similarity is registered in a broadened range extended to the dissipation scales $[2,13]$. A generalized scale invariance (extended self-similarity ESS) was proposed in [13], and then considered in the logPoisson model of turbulence $[3,4]$. Hidden statistical symmetries of the Navier-Stockes equations, hierarchy of moments, multifractality are behind the property of the ESS. The ESS infers a scaling $S_{q}(l) \sim S_{3}(l)^{\zeta(q) / \zeta(3)}$ for the extended range $l \geq 5 \eta$. All data from fusion devices, that we analyzed, demonstrate such ESS property (linear behavior in Fig. 3 ) over the time scales $\sim 1$ msec substantially longer than an inertial range in Fig. 2. The ESS corresponds to the considering of the scaling in a turbulent cascade not with respect to the usual distance, but with respect to an effective scale defined by the third order moment of the velocity field. 


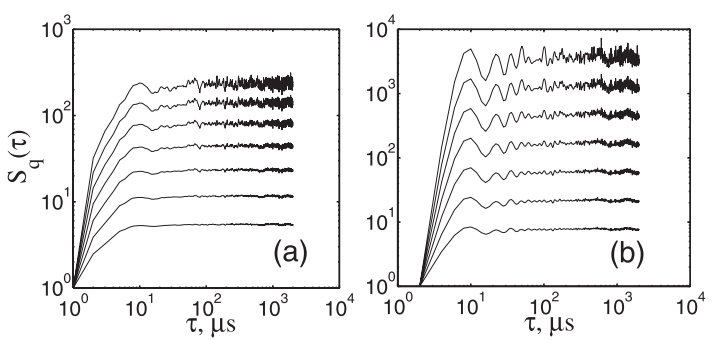

Fig. 2 The structure functions $S_{q}(\tau)$ of high orders $(q=2 \sim 8$ from bottom to top) vs. time scale $\tau$. (a) LHD divertor probe \#10 (b) LFS SOL JT-60U, shot\#44421.
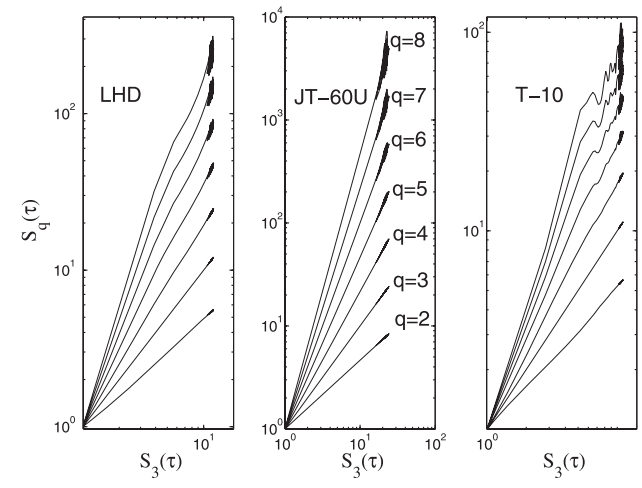

Fig. 3 ESS plot of the structure function $S_{q}(\tau)$ of high orders ( $q=2 \sim 8$ from bottom to top) from the third-order one. (a) LHD divertor probe, (b) LFS SOL JT-60U, distance from separatrix $42 \mathrm{~mm}$ (c) SOL T-10.

\section{The log-Poisson Model of the Tur- bulence}

The scaling of the third-order moment can be deduced analytically $(\zeta(3)=1)$, therefore scaling of $\zeta(q) / \zeta(3)$ can be analyzed in experiments to improve the precision of the scaling estimation, especially at moderate Reynolds numbers assessed in experiments. It allows to obtain more accurate values of $\zeta(q)$ by using a property of the ESS plotting $S_{q}$ as a function of $S_{3}$, Fig. 3. We treat experimental scalings in the frame of log-Poisson turbulence model [4] predicted a scaling:

$$
\zeta(q)=(1-\Delta) \frac{q}{3}+\frac{\Delta}{1-\beta}\left[1-\beta^{q / 3}\right] .
$$

It is based on the hypotheses of a "hidden symmetry" and a hierarchical structure of the moments of the energy dissipation. The logarithm of energy dissipation obeys the Poisson statistics (so-called the log-Poisson statistics) characterized by special scale-covariance properties. A hidden symmetry can be interpreted as a generalized scale covariance and $\beta$ is a characteristic of the intermittency of the energy dissipation $(\beta=1$ for non-intermittent fully developed turbulence). The quantity $\varepsilon_{l}^{\infty}$ (associated with the most intermittent dissipative structures) has a divergent scaling $\varepsilon_{l}^{\infty} \sim l^{-\Delta}$, as $l \rightarrow 0$, where $\Delta$ is a parameter depending on the dimension of the dissipative structure. In an
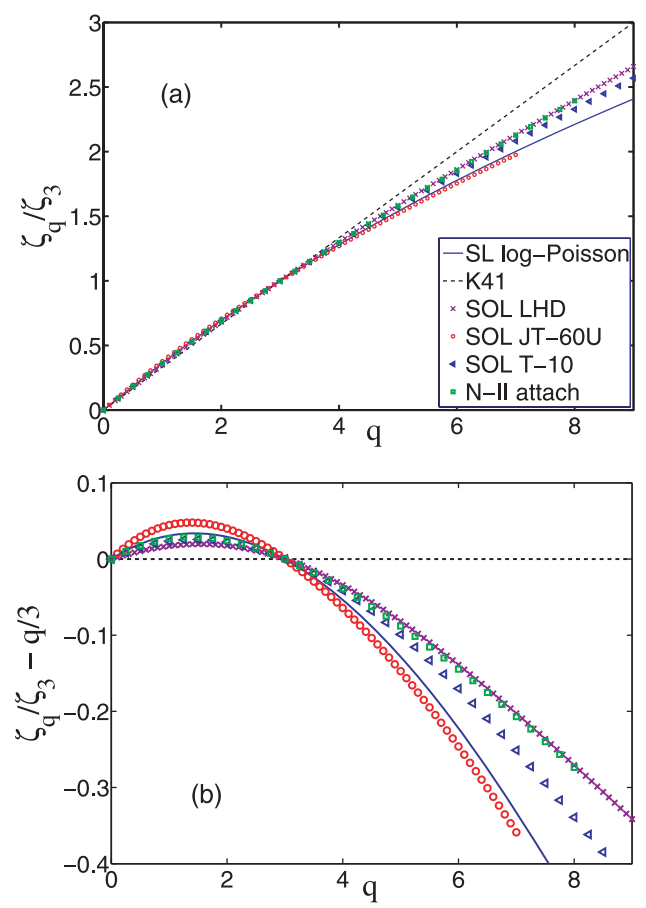

Fig. 4 (a) Structure function scaling vs. order q. Kolmogorov K41 (a dashed line) and She-Leveque (SL) log-Poisson (a solid line) models and (b) the same for a departure of the scaling from the K41.

isotropic 3D turbulence $\Delta=\beta=2 / 3$ (She-Leveque logPoisson model) which is obtained if the most dissipative structures are filaments. The ESS property is involved in the log-Poisson model. We use the wavelet transform modulus maxima method (WTMM) [5] to estimate $\zeta(q)$ from experimental signals. In Fig. 4 , the scalings $\zeta(q) / \zeta(3)$ are shown in the same plot with the scalings predicted by the K41 and the log-Poisson models. The scalings are anomalously deviated from the K41 scaling, Fig. 4b. Each experimental scaling could be fitted by (1) with adjusted parameters $\Delta$ and $\beta$. A solving of non-linear least-squares problem of fitting to (1) gives indexes in the range $\Delta=0.15 \sim 0.8$, $\beta=0.25 \sim 0.7$. Some signals have non-intermittent behaviour $(\beta=1)$. The observed range of $\Delta$ (between $1 / 3$ and 2/3) can be interpreted [14] that the most intermittent dissipative structures are one-dimensional filament structures in these cases. Such dissipative structures have most likely not a trivial geometrical topology but a fractal one.

The log-Poisson model of 3D turbulence was modified in MHD case to account for the IK phenomenology [7]. To test IK model we plot in Fig. 5 a deviation of relative exponents $\zeta(q) / \zeta(4)$ from IK scaling $q / 4$. The scaling of data in the vicinity of X-point in JT-60U is close to the IK indicating strong MHD turbulence property. The data from SOL are deviated strongly from IK scaling (see [6]). At the same time they are not fitted by the scaling for IK MHD log-Poisson model [7] :

$$
\zeta(q)=\frac{q}{8}+1-\left(\frac{1}{2}\right)^{q / 3} .
$$




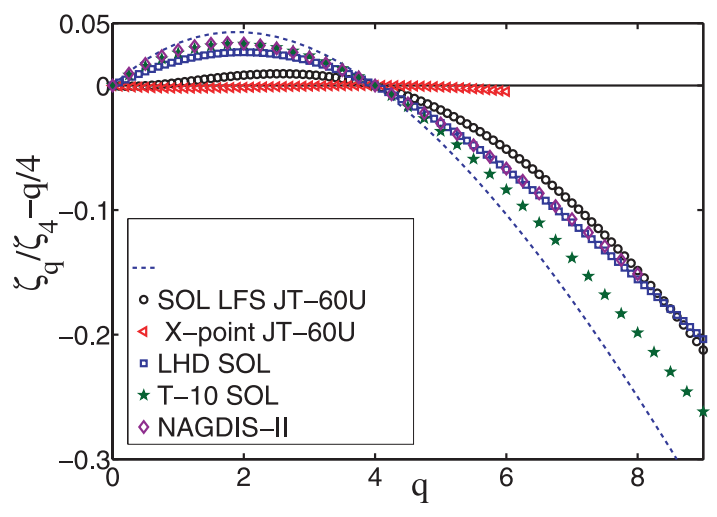

Fig. 5 Deviation of scaling from Iroshnikov-Kraichnan scaling $q / 4$ (solid line). IK MHD log-Poisson scaling (dashed line). Scaling in the vicinity of X-point JT-60U is close to IK.

It can be interpreted that IK phenomenology (twodimension strong anisotropy) is not available for a treatment of the SOL plasma turbulence.

\section{Transport Scaling Laws}

The statistical description of transport processes in fusion plasmas is an alternative approach to the traditional characterization of a transport based on the computation of effective transport diffusion coefficients assuming Einstein law of the diffusion. Traditionally, a displacement of particles across a magnetic field with time $\tau$ is considered as $\left\langle\delta x^{2}\right\rangle \propto D \tau$ with a constant (or independent on time scale) diffusion coefficient $D$. In these models, $D$ is estimated from some assumptions supposing some dominant physical mechanism and eliminating other contributions. In such approach, effects of a non-linear coupling of modes with different scales (large and small) is excluded that could lead to a significant deviation of the model properties from the real plasma properties. It relates especially to the SOL plasmas where many mechanisms (such as pressure gradient driven instabilities, stochastic magnetic structure, flow shearing, neutral drag, radiation instabilities, effect of the boundaries, etc.) can contribute to the transport process. Statistical approach is based on a general consideration of turbulent fluctuations suggesting some universal properties of intermittent turbulence. It allows skipping a consideration of concrete mechanisms driven the turbulent and transport process. Because a plasma transport in edge of fusion devices is a multi-scale process consisting of events of different scales (e.g., large scale blobs, and small scale coherent events, etc.), the statistical approach is most appropriative for a self-consistent description of turbulence and transport phenomena.

The log-Poisson model could be used to estimate a transport scaling based on the self-similarity indexes $\beta$ and $\Delta$ (Eq. (1)) that responsible for percolation effect in the turbulence. In a simplified approach [14], the diffusion scaling depends on the structure function scaling as $D \propto \tau^{K(-1)}$,
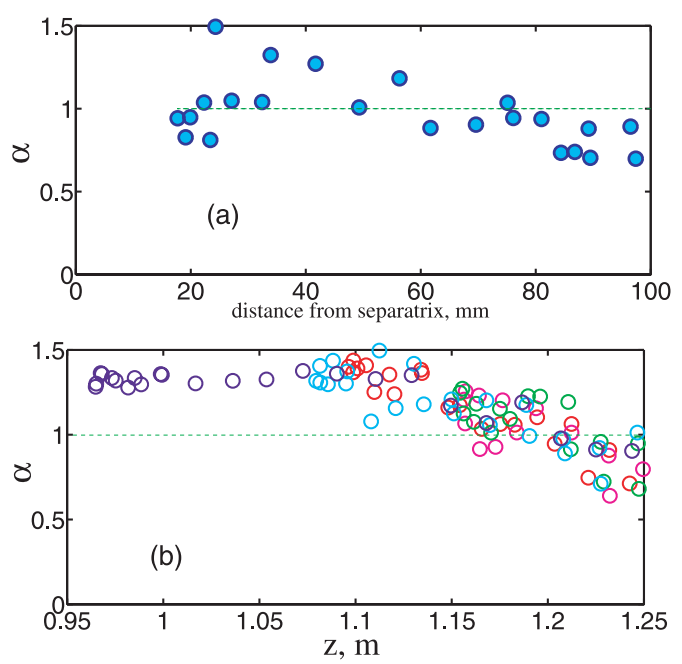

Fig. 6 Diffusion scaling index (a) JT-60U LFS SOL (shot\# 44421) (b) SOL LHD high $\beta$ shots vs. vertical coordinate $z, B=0.425 \mathrm{~T}$, fast scanning probe data.

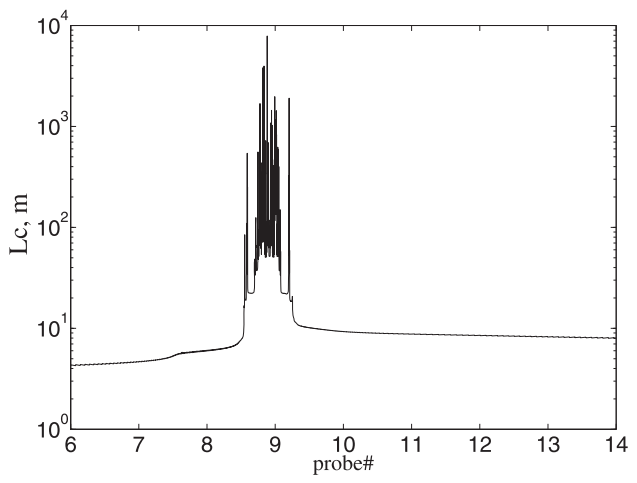

(a)

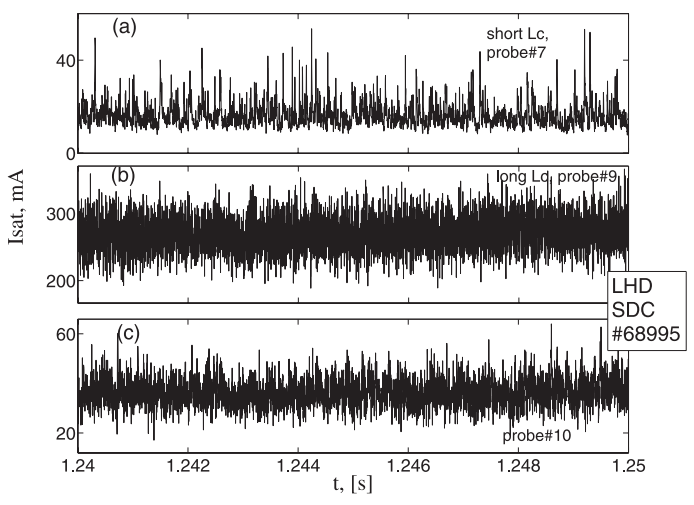

(b)

Fig. 7 (a) Connection length $L_{\mathrm{c}}$ profile on the divertor probes plane regarding the probes numbering. (b) Signals of $I_{\text {sat }}$ on divertor probes in LHD SDC shot \#68995.

the exponent $K(q)$ relates with the scaling of the high-order structure function $\zeta(q)$ as $K(q)=q-\zeta(3 q)$. A displacement of particles across a magnetic field with time $\tau$ is scaled as $\left\langle\delta x^{2}\right\rangle \propto D \tau \propto \tau^{\alpha}$ with an exponent $\alpha \propto 1+K(-1)$. This index was estimated from experimental scalings. It varies with a radius in JT-60U SOL (Fig. 6a). At radial distance $20-50 \mathrm{~mm}$, scaling exponents $\alpha$ exhibit a scattering 
behaviour between 1 and 1.5. This area is characterized by a specific behaviour of the SOL parameters [10].

In LHD SOL (Fig. 6b) the superdiffusion process $(\alpha>1)$ is observed at vertical distance $0.97-1.15 \mathrm{~m}$ in high $\beta$ shots.

In LHD the natural island layers overlap and the stochastic field structure (a natural helical divertor) appears between the LCFS and the residual X-point. The magnetic field line with large connection length $L_{\mathrm{c}}$ reaches the ergodic layer surrounding the core plasma region [15]. $L_{\mathrm{c}}$ varies from less than a few meters to over a few kilometers (Fig. 7a). The probe connected to the field line with a large $L_{\mathrm{c}}$ has a large ion particle flux (Fig. 7b). Statistical properties of the signals show a dependence on the connection length $L_{\mathrm{c}}$ (Fig. 7, 8). Ion particle flux to the divertor
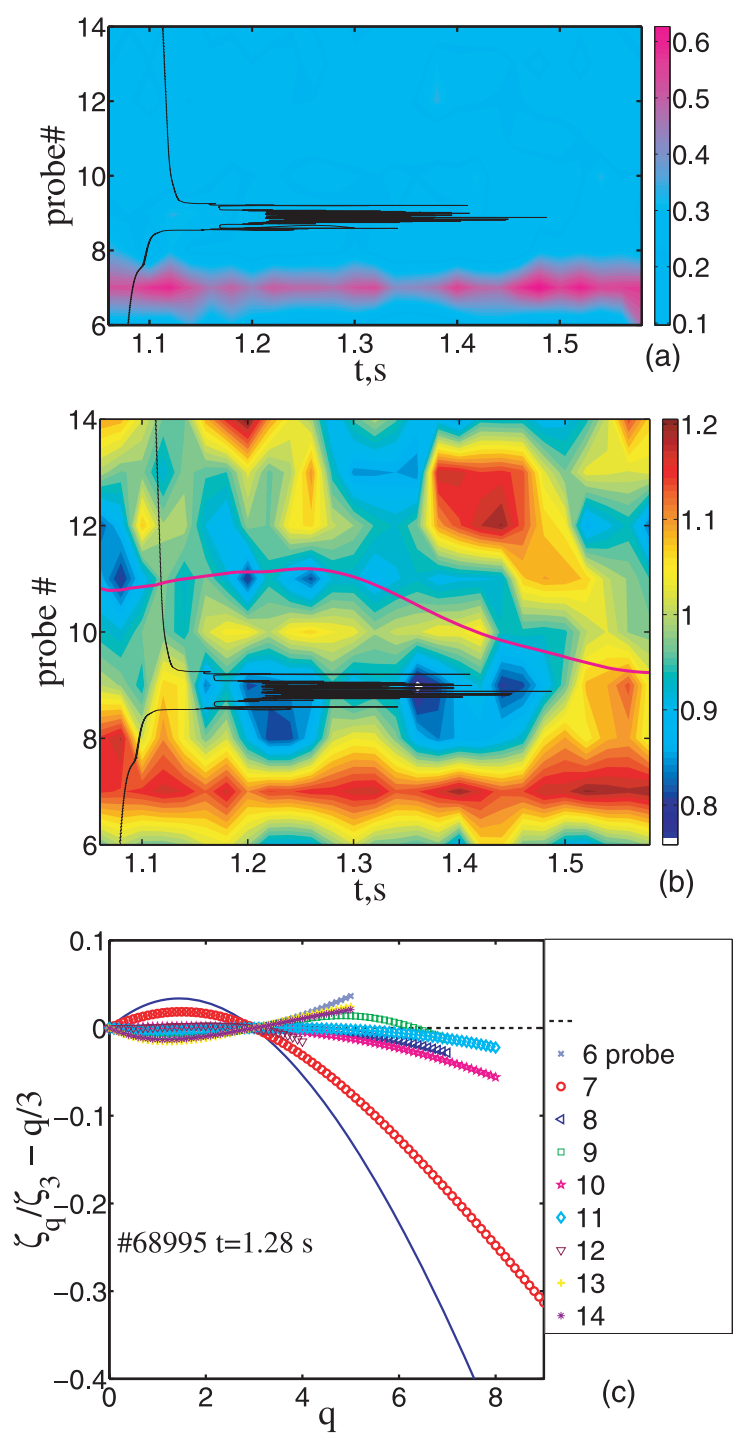

Fig. 8 LHD divertor probes data, shot \#68995 with SDC. (a) Multifractality level, (b) Diffusion scaling index $\alpha$, Magnetic connection length $L_{\mathrm{c}}$ (logarithm of magnitude, a.u.) in black line, and plasma storage energy evolution (magenta) (c) Structure function scalings, deviation from K41 (dashed line), She-Leveque (SL) log-Poisson scaling (line). plates [9] and multifractality level (deviation from Gaussian statistics, see a definition in [5]) follows the deposition profile of the magnetic field lines, Fig. 8a. In LHD, an increasing of $\alpha>1$ was observed in domains that are characterized by short connection length $L_{\mathrm{c}}$. Especially, this characterization is detected by probe \#7 (Fig. 8b) where the connection length $L_{\mathrm{c}}$ is less than $4 \mathrm{~m}$. The log-Poisson scaling characterizes a specific behaviour of \#7 probe signal (Fig. 8c). Other domains are closer to the K41 scaling (Fig. 8c). Minimal exponent $\alpha$ is observed at the domain related to the longest $L_{\mathrm{c}}$ (probe \#9, Fig. 8b). This property is kept even at discharge evolution at super-dense core (SDC).

The intermittency properties don't depend on density (Fig. 9): the substantial increasing of the plasma density at IDB phase don't lead to a detectable change of statistical property. We have investigated the scaling at different discharge periods corresponded to different plasma density at the probe location (Fig. 9a). At ITB phase, a change of pressure gradient in the edge may lead to a reconstruction of the turbulence structure. Amplitudes of fluctuations are changed (Fig. 9a) substantially. But the statistical property of fluctuations in the domain related to the long $L_{\mathrm{c}}$ does not

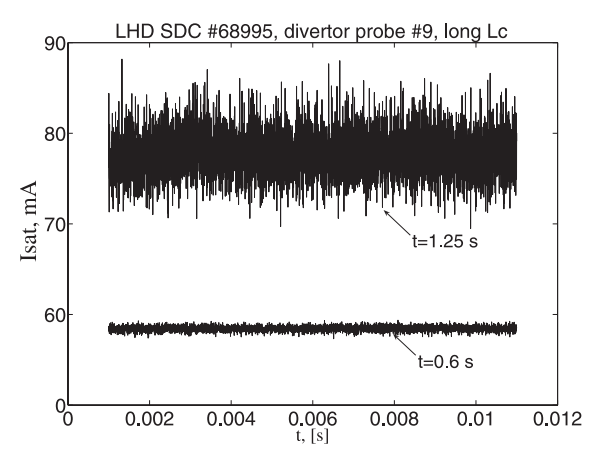

(a)

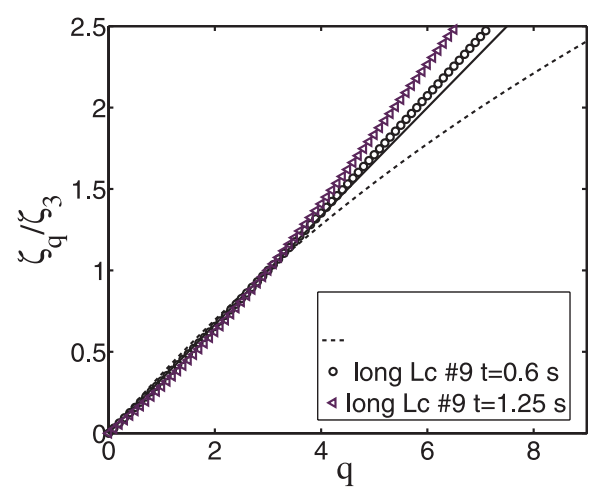

(b)

Fig. 9 The substantial increasing of the plasma density at LHD IDB phase (a) keeps the scaling close to $\mathrm{K} 41$ (b), $I_{\text {sat }}$ on probe \#9 at long $L_{\mathrm{c}}$. The K41(line) and She-Leveque logPoisson (dashed line) scalings are shown for the reference. 


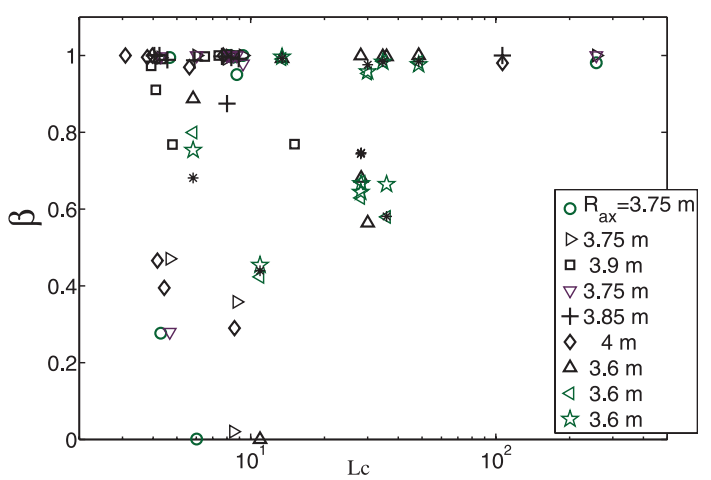

Fig. 10 Intermittency characterization $\beta$ vs. connection magnetic length $L_{\mathrm{c}}$ on divertor probes. $\beta=1$ corresponds to a non-intermittent behaviour. Intermittency is degraded at large $L_{\mathrm{c}}$. Divertor probe measurements on LHD in shots (\#\# 68955, 68995, 68996, 69357, 70146, 68921, 69261, $69268,69325,69360,69286)$ with different magnetic axis position $R_{\mathrm{ax}}=3.6 \mathrm{~m} \sim 4 \mathrm{~m}$ at ITB phase.

change. We detected this property related to the domain associated with the long $L_{\mathrm{c}}$. It demonstrates a preservation of the scaling close to the K41 (Fig. 9b).

We investigated a dependence of intermittency level in the series of LHD experiments with the different magnetic topology at different magnetic axis position $R_{\mathrm{ax}}$ (from $3.6 \mathrm{~m}$ to $4 \mathrm{~m}$ ). The parameter $\beta$ characterized intermittency (Eq. (1)) is estimated from the analysis of a stable period of discharges at super dense core phase with minimal plasma current (less than $5 \mathrm{kA}$ ). It was observed a degradation of the intermittency at large $L_{\mathrm{c}}$ (Fig. 10). At short $L_{\mathrm{c}}$ a tendency of parameter to be in the range $0<\beta<1$ is observed, that is a case of the intermittent turbulence. We stress that this result is obtained in the series of experiments with different magnetic topology and edge plasma profiles. Despite a scattering data for the short $L_{\mathrm{c}}$, a clear degradation for the long $L_{\mathrm{c}}$ could be interpreted in favor of hypothesis considered a dependence of the intermittency property on a magnetic connection length. The study of this effect should be continued in the future.

\section{Conclusions}

In conclusion, the statistical properties of the intermittent turbulence show a striking empirical similarity in the SOL plasma region of fusion devices. Scalings of the structure functions strongly deviate from the Kolmogorov's K41 theory prediction. The anomalous behavior of scaling is similar in the SOL plasma of helical device, tokamaks, and linear machine. Experimental scalings are close to the log-Poisson model. One-dimension filament structures are likely the most intermittent dissipative structures. The similar behavior of the scalings has been observed in the edge of fusion devices with different magnetic topology and heating. It supports a view that the edge plasma turbulence displays universality. By using self-similarity indexes, transport scaling indexes are estimated from percolation property of the turbulence with non-trivial self-similarity. The results of our study improve our understanding of intermittent turbulence in the edge of fusion devices.

\section{Acknowledgements}

This work was supported by the NIFS budged NIFS06ULPP511, JT-60U budged, the Rosatom RF. V.P.B. thanks NIFS for hospitality.

[1] A.N. Kolmogorov, Dokl. Akad. Nauk SSSR 30, 299 (1941) ( Proc. R. Soc. London, Ser. A 434, 9 (1991)).

[2] U. Frish, Turbulence: The Legacy of A.N. Kolmogorov (Cambridge : Cambridge University, 1995).

[3] Z.S. She and E. Leveque, Phys. Rev. Let. 72, 336 (1994).

[4] B. Dubrulle, Phys. Rev. Lett. 73, 959 (1994).

[5] V.P. Budaev et al., Nucl. Fusion 46, S181 (2006).

[6] W. Müller and D. Biskamp, Phys. Rev. Let. 84, 475 (2000).

[7] H. Politano and A. Pouquet, Phys. Rev. E 52, 636 (1995).

[8] P. Goldreich and S. Sridhar, Astrophys. J. 438, 763 (1995).

[9] S. Masuzaki et al., Nucl. Fusion 42, 750 (2002).

[10] Asakura et al., Nucl. Fusion 44, 503 (2004).

[11] N. Ohno et al., Nucl. Fusion 41, 1055 (2001).

[12] A.S. Monin and A.M. Yaglom, Statistical Fluid Mechanics (MIT Press, Cambridge, MA, 1975).

[13] R. Benzi et al., Phys. Rev. E 48, R29 (1993).

[14] V.P. Budaev et al., Contrib. Plasma Phys. (to be published).

[15] T. Morisaki et al., J. Nucl. Mat. 548, 313 (2003). 